\title{
AN ELECTRICAL IMPEDANCE TOMOGRAPHY SYSTEM FOR THYROID GLAND WITH A TINY ELECTRODE ARRAY
}

\author{
Moustafa Ahmed ${ }^{1}$, Yara Mouhamed ${ }^{2}$, Nivan M. Fikry ${ }^{3}$, Hesham Ali Badawi ${ }^{3}$, \\ Mohamed A. Bondok ${ }^{4}$ and Mona S. El-Neklawi ${ }^{4}$ \\ ${ }^{1}$ Faculty of Allied Medical Science, Pharos University in Alexandria, ${ }^{2}$ Faculty of \\ Medicine, Alexandria University, ${ }^{3}$ Medical Research Institute, Alexandria University, \\ Faculty of Applied Medical Science, October 6 University, Egypt
}

\begin{abstract}
Electrical impedance Tomography (EIT) is a non-invasive imaging technique based on measuring of the electrical conductivity and capacitance of abnormal and normal human tissues. The present work aims to develop an EIT imaging system for imaging thyroid gland. Patients with thyroid nodules were eligible for the study. The study was conducted on two groups of participants: control group consists of 20 normal female cases and experimental consists of 20 goiter female patients. The thyroid nodule location, size, and type measured by ultrasound. Thyroid gland conductivity and permittivity were recorded using EIT. The impedance measurement is done through the applying of two probes: one probe to the neck region (scanning probe) and the rest region (reference probe) with electrolytic gel for each probe, then the system software proceeds to reconstruct the image and calculate the electrical impedance of the thyroid gland on a personal computer which acts as an output display and storage for case information. The thyroid scanning probe has 64 electrodes embedded on a small space (30 mm diameter and $50 \mathrm{~mm}$ height) inside of the probe. Multifrequency impedance measurements are typically made by applying an electric current to a target mass by using of the scanning probe and measuring the developed voltage. The present EIT system provides real- time visualization of the spatial distribution of the electrical properties of the thyroid tissue. Images obtained from the bioimpedance (BI) were compared to images obtained from the ultrasound imaging, results showed great similarity between the two diagnostic images. Tumor tissue has higher resistance and capacitance value than that of normal thyroid gland.
\end{abstract}

\section{KEYWORDS}

Electrical impedance Tomography, Electrical properties of the thyroid tissue Thyroid gland imaging,

\section{INTRODUCTION}

During the past decades, an increasing of thyroid cancer has been reported.[1] Electrical impedancetomography (EIT) is an imaging method has wide practical applications in medicine. It produces an image of the cross-sectional impedance within an object by applying currents while measuring the corresponding voltages at the boundary [2]. EIT is different from other image modalities in clinics. This technique can provide additional information on tissues under diagnosis. Also, EIT offers the merits of low cost, no ionizing radiation, and noninvasiveness. Several research groups have published reports about the EIT hardware and methods [3,4]. EIT is 
a frequency measuring system provide useful information about the electrical properties of tissues and cells (5) and have possible application in several areas [6]. There are several EIT practical limitations such as the complicated spatial distribution of the bio-impedance that arises from complex structure of biological tissues, in addition to the structure and arrangement of measurement electrodes. To obtain reasonable images, at least several thousand of measurements are usually carried out. These results take measuring and analyzing time. Therefore, it is difficult to achieve high precision and to assert measurement results as clinically relevant information. In order to overcome this drawback, there is a need to address several issues for employing EIT in medical application such as, estimating impedance parameters for local tissue and the shape of electrode structure.[7] The most important drawback of the EIT is its poor image resolution which is often restricted by number of electrodes. The present study overcome this drawback by increasing number of electrodes, which transmit the electrical current through thyroid tissue to measure the electrical characteristics of the thyroid gland to be able to differentiate between normal and abnormal tissues in it.

\section{Materials and Methods}

In the present study portable thyroid gland electrical impedance tomography instrument (Figure 1) is designed and constructed. In this instrument, multifrequency bioimpedance measurements in the range of $5 \mathrm{KHz}$ to $1 \mathrm{MHz}$ are typically made by applying an electric current $(0.5-\mathrm{m} 4 \mathrm{~mA}$ RMS) to a target mass using a square matrix of 64 electrodes and measuring the developed voltages between matrix electrodes and a reference electrode. This instrument interfaced to a personal computer using a microcontroller to facilitate measurements processing at different frequencies rapidly. The electrical measurements require the placement of a conducting gel on the scanning probe and on the reference electrode. The reference metal electrode was held in the seated patient's hand. The thyroid gland electrical impedance Tomography (TGIT) system used in the present work consists of: microcontroller, analog to digital convertor (ADC), divider circuits, eight 8-channelds multiplexers, peak detector system and two probes (scanning probe and reference) electrode. Personal computer (PC) was used to control operations, provides image reconstruction and visualizes it for operator on screen.

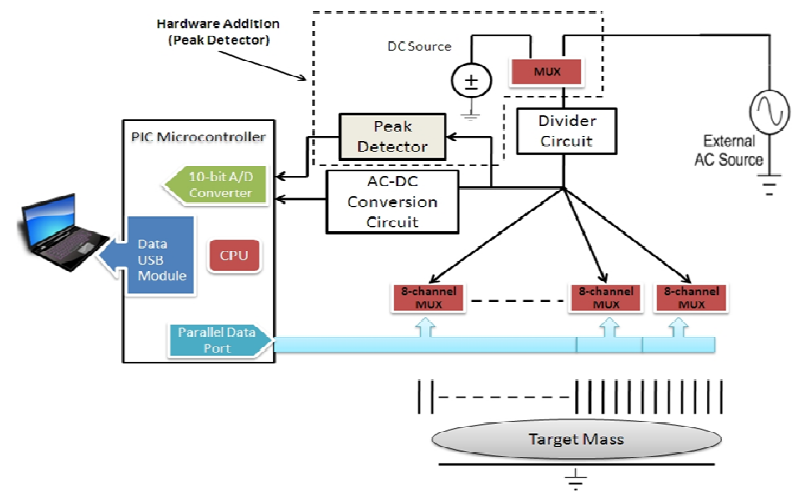

Figure 1: Block diagram of thyroid gland impedance Tomography system hardware 
The thyroid scan probe has the measuring array of $64 \mathrm{Ag}-\mathrm{AgCl}$ electrodes. The main part of functions is maintained by the microcontroller: Analog to digital converters for current, and voltage, signal generation and quadrate calculation. The microcontroller generates the voltage signal through a small value resistor between an active electrode of the 64 electrodes array which is choose by the multiplexer and virtual ground electrode. Current flows through the patient body from one of the active electrodes to the reference electrodes. Injected current is measured on the small resistor by the the first Analog to digital Converter (ADC!) of the microcontroller. The microcontroller measures voltage and current data set quadratures from 5 $\mathrm{kHz}$ up to $1 \mathrm{MHz}$ for all electrodes in the array using MUX OUT and ADC2 and transfers data set to the PC which reconstructs image and shows contact map three depths $2,5,8 \mathrm{~mm}$ on screen for 10 and $50 \mathrm{kHz}$.

Scanning circular probe which is $3 \mathrm{~cm}$ in diameter consisting of 64 silver- silver chloride (Ag$\mathrm{AgCl}$ ) was constructed. Each silver electrode is $1 \mathrm{~mm}$ in diameter arranged in an $8 \times 8$ matrix; they are fixed in headers to keep the arranged matrix in their order and also offered an isolation space of $2 \mathrm{~mm}$ between each silver electrode. The probe structure is then embedded in a mixture of $10 \mathrm{ml}$ araldite, $10 \mathrm{ml}$ of dodecenyl succinic anhydride (DDSA), and 4 drops per $5 \mathrm{ml}$ of benzyl dimethylamine BDMA (accelerator). The mixture is placed in oven for 24 hours at temperature of $60^{\circ} \mathrm{C}$ for polymerization as shown in figures $2(\mathrm{~A} \& \mathrm{~B})$

Reference probe is placed on the wrest of the same side of the measured thyroid lobe, for example it will be placed on the right wrest when measuring the right thyroid lobe. It is a clamped shape. Electrolytic gel is applied to both probes in the scanning process to ensure the best conductivity.

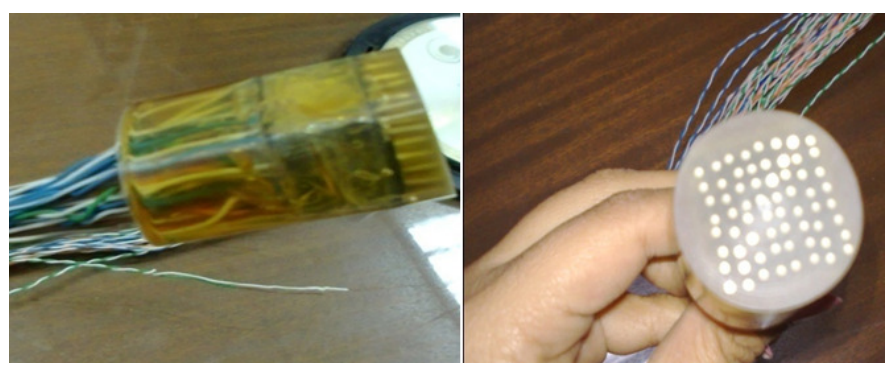

(a)

(b)

Figure 2: Scanning probe. (a) top view showing silver electrodes. (b) side view of the probe and the connecting wires.

\section{System software}

Bioimpedance instrument (Figure 3) used in the present study operates through two operational modes: Image mode acts as a scanning system based on the bioelectrical characteristics of a target mass. Multi-frequency contrast is used to generate a tomographic image for the scanned mass and R-C mode which has the ability to measure both the resistance and the capacitance of the target mass. Image mode mechanism is basically split into two subsystems; data acquisition system and image reconstruction system. The digital value of the voltage across each electrode is transmitted via the built-in USB module. This digital data is used to generate the tomographic image on the target computer. Image reconstruction system is responsible for: Ordering of electrode set data elements, Performing 2D linear data interpolation between basic data elements, 
The developed software is split into two main interconnected components:

1 - Data acquisition software (namely: Bio-Image Scanner V2.0) was developed using (Microsoft Visual Basic .NET) to: Control the hardware data acquisition system via the computer's USB HID (Human Interface Device) and collect and store data elements corresponding to each member of the electrode set. When the data stream ends, the software starts generating image.

2 - Image generating software (namely: plot_impedance.exe) was developed using (MATLAB 7.5) to reorder imported data elements, perform linear data interpolation and generate a tomographic image using a predefined color code.

\section{Dielectric measurements:}

A 5 DC volt generated from the power supply circuit is applied to the electrode system. Using the peak detector circuit, the values of the equivalent resistance $(\mathrm{R})$ and capacitance $(\mathrm{C})$ is calculated for each electrode. Both $\mathrm{R}$ and $\mathrm{C}$ calculated values are used to calculate the Reactance $(\mathrm{Xc})$ and Impedance $(\mathrm{Z})$.The measured values were used to calculate permittivity $\dot{\varepsilon}$ and conductivity $\sigma$ of the thyroid tissue.

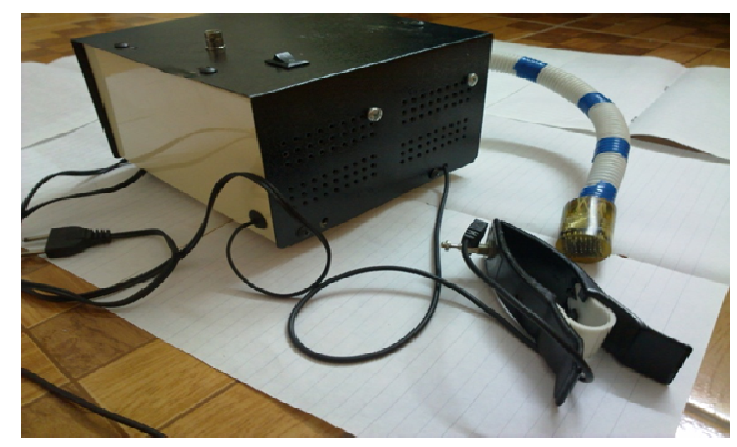

\section{RESULTS}

Figure 3: Bioimpedance instrument

To ensure the accuracy of the readings and images obtained from the new bioimpedance instrument, a second diagnostic method was applied using ultrasound. Both methods were applied to image the neck of 20 goitre patients with its different stages and 20 normal female cases (control) of 40-50 years of age. Written consent was taken from the participant persons following a protocol approved by ethics commute of Medical Research Institute, Alexandria University. The data acquisition time was approximately 15 seconds for each scan, with an image reconstruction time of 32 seconds and 48 seconds for generating the desired parameters. Several calculations were obtained leading to enumerous electrical identification of the thyroid tissue and its abnormalities. Abnormal tissues exhibit sharply different bioelectrical properties compared with the normal tissues. In an early stage of tumor tissue, abnormalities start to develop in cellular water/electrolyte content, cell membrane permeability, and orientation/packing density of cells. these changes introduce bioimpedance abnormalities that can be detected by using this suggested bioimpedance instrument. 


\section{A. Image reconstruction of thyroid tissues:-}

Normal thyroid tissues: Images obtained using BI and ultrasonography show a great similarity (Figure 4)

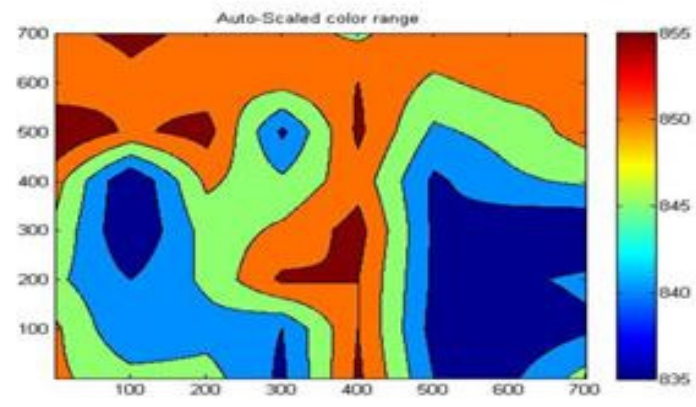

(a)

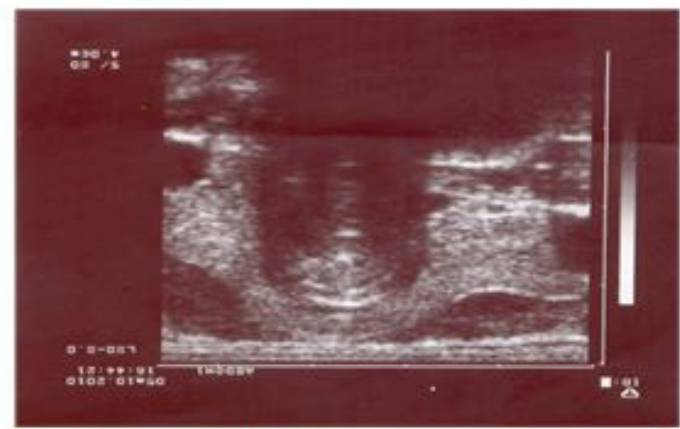

(b)

Thyroid Tumor

Figure 4: Normal tissue of thyroid gland. (a) BI image. (b) US image.

Images obtained from a woman with thyroid tumor using BI instrument and ultrasonography using an HDI 3000 ATL ultrasound machine are shown in Figure 5. The images indicated the presence of sizable all defined, nodule in right thyroid lobe with irregular bounders. In the BI image (figure 5a), the orange to dark red regions resembles a calcified tissue in the thyroid gland which is similar in shape to that present in the ultrasound image (figure $5 b$ ).

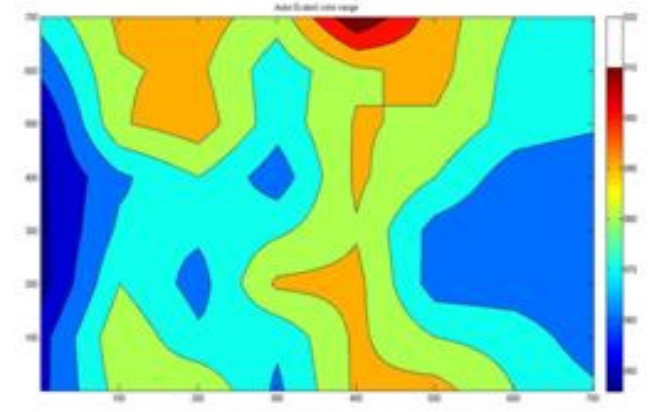

(a)

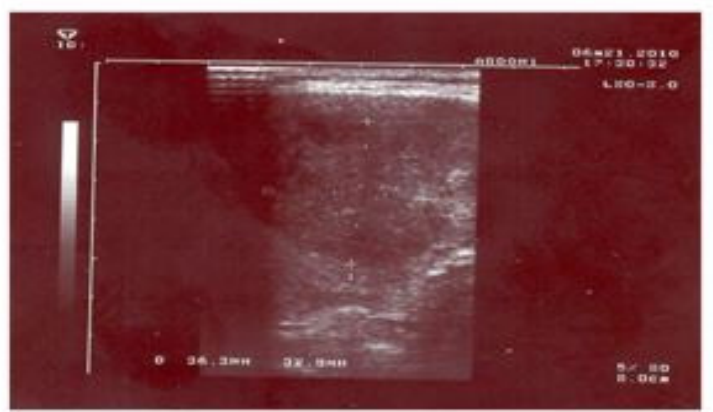

(b)

Figure 5: Image of thyroid gland suspicious nodule. (a) BI image. (b) US image.

Active thyroid gland

These images show active thyroid gland (figure 6) forming a calcified nodule in the left thyroid lob

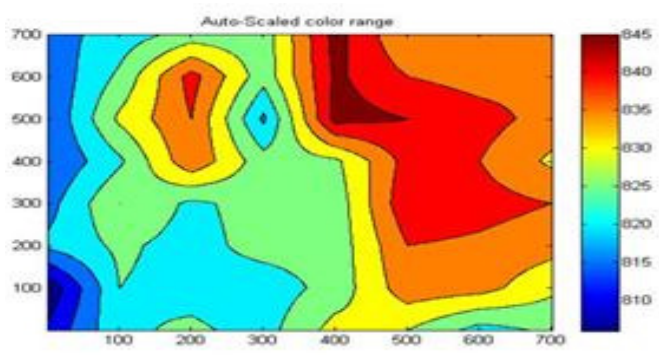

(a)

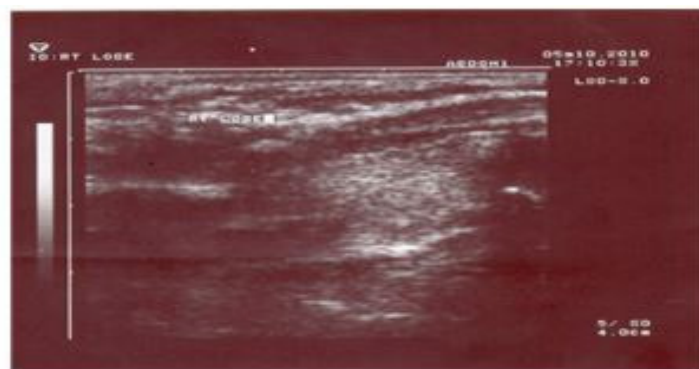

(b)

Figure 6: Calcified nodule in thyroid gland. (a) BI image. (b) US image. 
Figure 7 shows images of the left thyroid lobe which is mildly enlarged showing multiple variable sized nodules

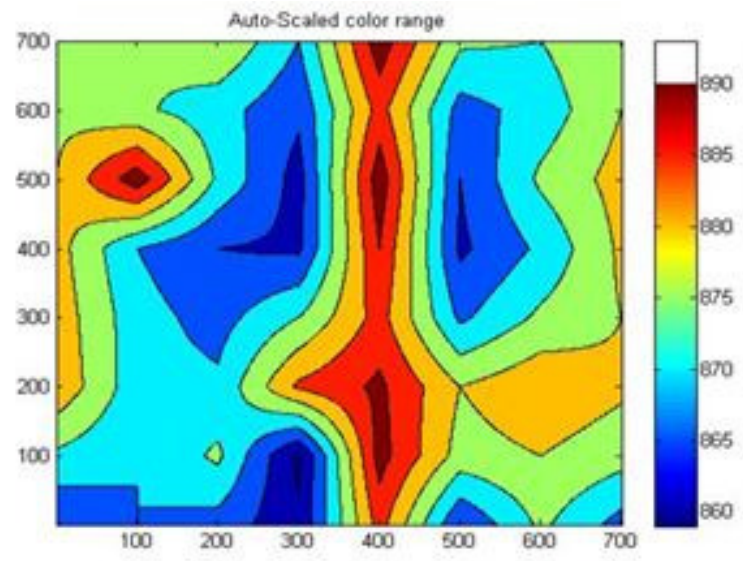

(a)

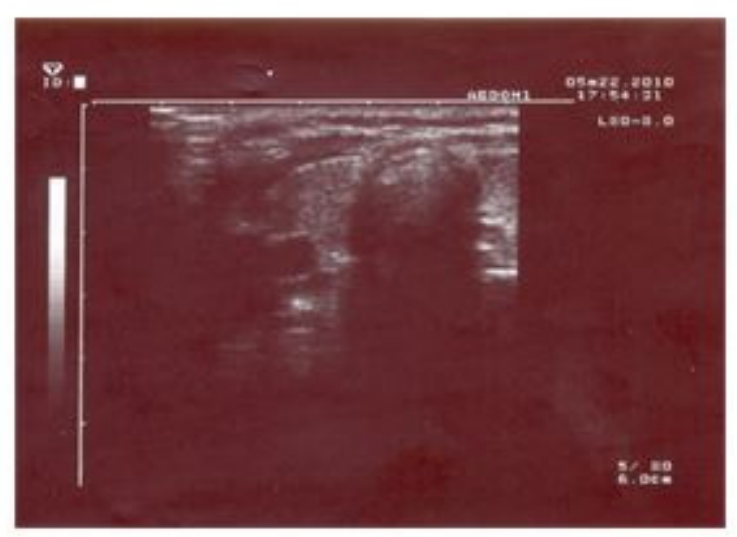

(b)

Figure 7: Enlarged active thyroid left lobe. (a) BI image. (b) US image.

\section{B. Characterization of thyroid tissues}

Multi-frequency range from $5000 \mathrm{~Hz}$ up to $1 \mathrm{MHz}$ electrical properties of a specific area of both control and tumor tissues in the thyroid gland were measured for the quantitative analysis of the obtained images, frequencies were entered manually from the function generator used. Measurements were repeated and calculated only for one point of the probe; the point selected is E5 for its centre location in the probe matrix.Since different tissues exhibit different bioelectrical characteristics, BI was able to differentiate between normal and abnormal tissues through measuring their resistance values. Results indicated that tumorous tissue has higher resistance than that of normal tissue with a wide range (Figure 8). Capacitance measurements show that capacitance values of tumor tissues is slightly higher than that of normal tissue (figure 9)

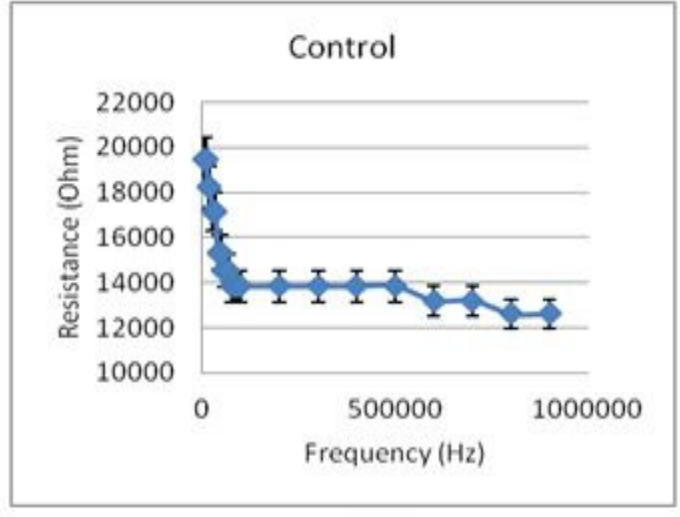

(a)

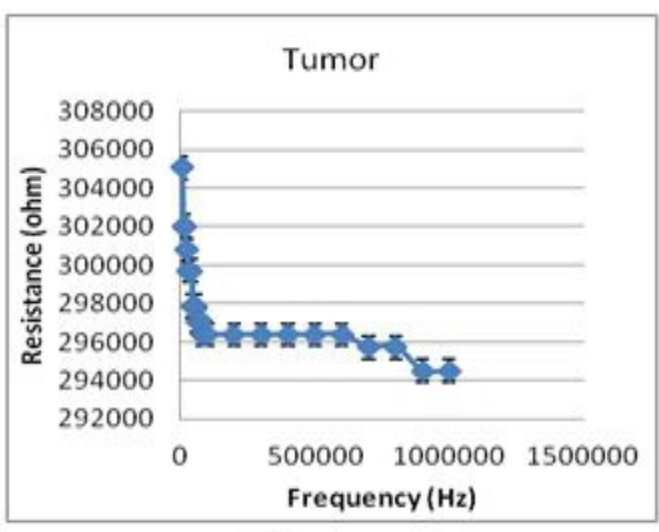

(b)

Figure 8: The thyroid resistance curve normal case abnormal case with tumor in thyroid tissue. 
International Journal of Biomedical Engineering and Science (IJBES), Vol. 3, No. 3, July 2016

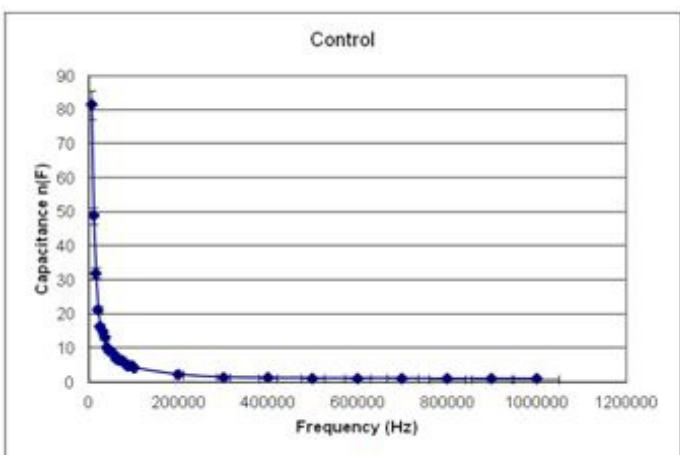

(a)

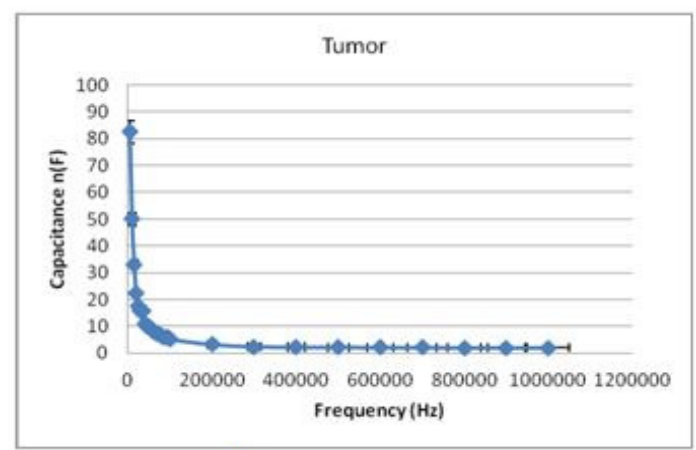

(b)

Figure 9: Thyroid capacitance (a) normal thyroid gland. (b) tumor in thyroid tissue.

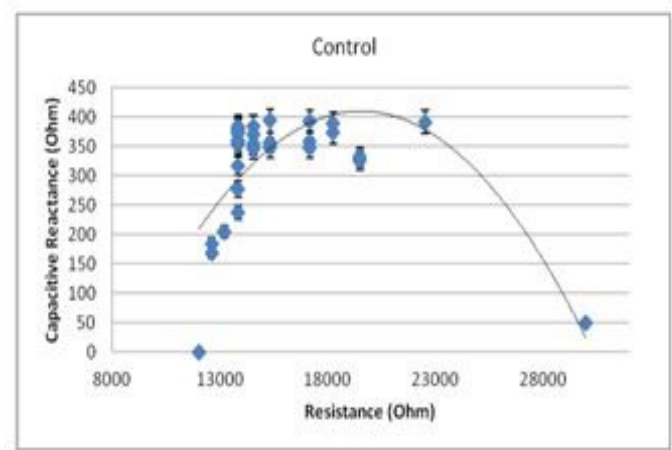

(a)

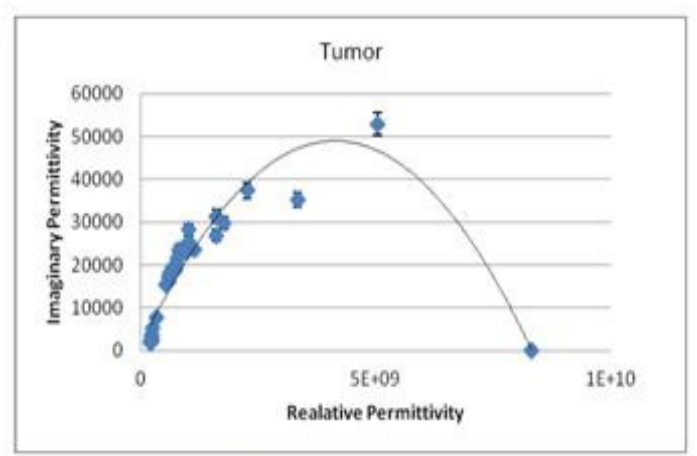

(b)

Figure 10 complex diargam between real and imaginary permittivity of (a) normal and (b) tumor thyroid tissues.

In figure 11 representation of the phase spectra $(\phi)$ of normal thyroid tissues proved that it is double the phase spectra of tumor tissue.

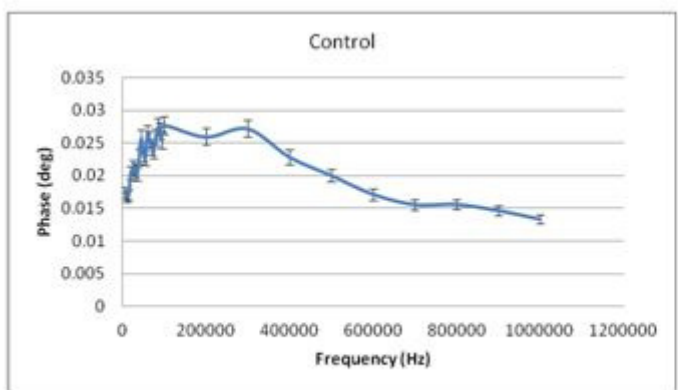

(a)

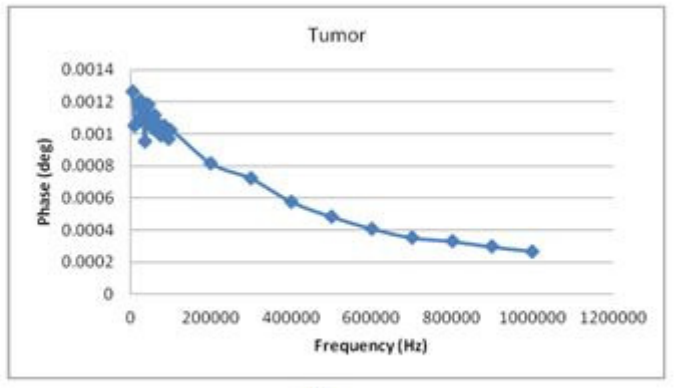

(b)

Figure 11: Phase spectra along frequency range from $5000 \mathrm{~Hz}$ to $1 \mathrm{MHz}$ of thyroid gland. (a) Phase spectra in case control tissue. (b) Phase spectra in abnormal tumorous patient. 


\section{DISCUSSION}

The study of human body composition in health and disease is of interest in both research and clinical practice. Every organ in the man's body posses its own electrical conductance characteristics. Accurate measurement of the impedance of bio-materials over a broad frequency range yields valuable information about the electrical properties of the material. The primary aim of this study was to develop an electrical bioimpedance tomography system for imaging thyroid gland. Bioimpedance is a non-destructive technique which makes use of low level electrical signals such as those which are already present in bio-materials. Accurate measurement of the impedance of bio-materials over a broad frequency range yields valuable information about the electrical properties of the material. The present study demonstrates that measurement of EIS signal may provide useful supplementary information to assist in classifying between malignant and benign thyroid nodules and lead to a reduction in the number of unnecessary thyroid surgeries. The results showed that bioimpedance measurements are able to differentiate between normal and abnormal tissues through measuring their resistance values. Images obtained using EIT imaging system and ultrasonography show a great similarity for both normal and tumor thyroid glands.

In the present work, design and construction of a home-made bioimpedance instrument was achieved to produce an image and give electrical information about the electrical characteristics of thyroid gland. The study was conducted on two groups of participants: group A (control) consisting of 20 normal cases and group B (experimental) consisting of 20 goiter patients in different stages. The BI measures is done through the applying of two probes to the neck region (scanning probe) and the rest region (reference probe) with electrolytic gel for each probe, then the system software proceeds to reconstruct the image and calculate the dielectric measurements on a personal computer which acts as an output display and storage for case information. This process takes place only if a function generator is connected to the $\mathrm{BI}$ to ensure the presence of required AC signal.

Images obtained from the BI were compared to images obtained from the ultrasound machine which was used as a reference diagnostic method, results showed great similarity between the two diagnostic images. The BI came with high resolution image due to the increase of number in the scanning probe up to 64 electrodes Measurements and calculations agreed with previous work and adopted the same concept that tumor tissue has higher resistance and capacitance value than that of normal thyroid gland.

The home made instrument is low in cost, easy to apply, portable, capable of on-line monitoring and it is safe to be used with no ionizing risk. Many parameters are measured and calculated lead to the following observations that undergoes conclusions of many previous work:

- There are significant differences between the electrical impedance of the cancerous and healthy tissues so EIS may consider a promise which could be used in differentiating of the thyroid nodules

- Bioelectrical impedance technique afford a fast, simple, accurate, valid and reliable method for thyroid gland imaging

- EIS hardware and software optimization could be improved upon the already favorable negative predictive value in indeterminate thyroid nodules. The results manifest how changes in the electrical properties of the thyroid gland tissues associated with the 
tissues abnormality:-

- Resistance of tumor tissue is much higher than resistance of normal thyroid gland, with clear significant difference, while the resistance of the calcified tissue and the nodules presented in the thyroid are slightly higher than normal resistance tissue

- Capacitance of tumor tissue, calcified tissue, nodules in the thyroid gland slightly differs from the capacitance value of the normal thyroid gland.

- Capacitive Reactance of tumor tissue is of slight difference below the capacitive reactance of normal thyroid tissue as it depends in its calculation on the same slight difference in capacitance.

- Relative to resistance observations conductance of normal tissue is much higher than conductance values of tumor tissue which is extremely low.

- Impedance of normal thyroid tissue is very low compared to impedance values calculated of tumor tissue, as it is of very high impedance value.

- The phase spectra of normal thyroid tissues proved to be double the phase spectra of tumor tissue.

- Conductivity of normal thyroid tissue is very high while conductivity of tumor tissue is very low.

- Realtive permittivity of tumor tissue increased with the increase in resistance and recorded its highest measurement for both tissues at the highest resistance value which was $305073 \Omega$ for the tumor tissue and $22530 \Omega$ for the normal tissue.

- Imaginary conductivity of normal thyroid gland didn't reach $3000 \mathrm{~S} / \mathrm{m}$ while the imaginary conductivity of the tumor tissue in the thyroid gland exceeded $5000 \mathrm{si} / \mathrm{m}$.

All the above measurements and calculations were estimated at multi-frequency range from 5000 $\mathrm{Hz}$ up to $1 \mathrm{MHz}$. The results of this study will serve as basis for future EIT studies. If these results are confirmed in large-scale trials, EIS may be consider as an important part of the evaluation of thyroid nodules.

\section{REFERENCES}

1. B. A. Kilfoy, T. Zheng, T. R. Holford, X. Han, M. H. Ward, A. Sjodin, Y. Zhang, Y. Bai, C. Zhu, G. L. Guo, N. Rothman, and Y. Zhang "International patterns and trends in thyroid cancer incidence", 19732002 Cancer Causes Control; 20(5): 525-531, 2009

2. D. Holder, "Electrical Impedance Tomography: Methods, History, and Applications", Taylor \& Francis, Basingstoke, 2005.

3. J. Wilson, P. Milnes, A. R. Waterworth, R. H. Smallwood, and B. H. Brown,"Mk3.5: a modular, multifrequency successor to the Mk3a EIS/EIT system" Physiol. Meas., Vol. 22, pp. 49-54, 2000

4. N. Liu, G. J. Saulnier, J. C. Newell, and T. J. Kao, "ACT4: "A High-precision, Multi-frequency Electrical Impedance Tomography," Proc. 6th Conf. on Biomedical Applications of EIT. London, 2005

5. Yufera, A. A. Rueda "Method for bioimpedance measure with four- and two-electrode sensor systems". Conf Proc IEEE Eng Med Biol Soc.; 2318S-21S, 2008

6. K. Norman , M. Pirlich, J. Sorensen , P. Christensen , M. Kemps , T. Schütz , et al. "Bioimpedance vector analysis as a measure of muscle function". Epub.; 28: 78S-82S, 2009.

7. S. Pandya, A. Arimoto, Ankur Agarwal \& Y. Kinouchi. "A Novel Approach for Measuring Electrical Impedance Tomography for Local Tissue with Artificial Intelligent Algorithm". International Journal of Biometrics and Bioinformatics, (IJBB), Volume (3): Issue (5), 65-81,2009 